Check for updates

Cite this: RSC Adv., 2018, 8, 5090

Received 4th August 2017

Accepted 16th January 2018

DOI: $10.1039 / \mathrm{c} 7 \mathrm{ra0} 8641 \mathrm{e}$

rsc.li/rsc-advances

\section{Design, synthesis, and characterization of lightly sulfonated multigraft acrylate-based copolymer superelastomers $\dagger$}

\author{
Konstantinos Misichronis, (D) ${ }^{\text {ab }}$ Weiyu Wang, ${ }^{c}$ Shiwang Cheng, ${ }^{b}$ Yangyang Wang, (D) ${ }^{c}$ \\ Umesh Shrestha, ${ }^{a}$ Mark Dadmun, (iD ab Jimmy W. Mays ${ }^{\text {ab }}$ and Tomonori Saito (iD) ${ }^{* b}$
}

\begin{abstract}
Multigraft copolymer superelastomers consisting of a poly( $n$-butyl acrylate) backbone and polystyrene side chains were synthesized and the viscoelastic properties of the non-sulfonated and sulfonated final materials were investigated using extensional rheology (SER3). The non-linear viscoelastic experiments revealed significantly increased true stresses (up to 10 times higher) after sulfonating only $2-3 \%$ of the copolymer while the materials maintained high elongation (<700\%). The linear viscoelastic experiments showed that the storage and loss modulus are increased by sulfonation and that the copolymers can be readily tuned and further improved by increasing the number of branching points and the molecular weight of the backbone. In this way, we show that by tuning not only the molecular characteristics of the multigraft copolymers but also their architecture and chemical interaction, we can acquire thermoplastic superelastomer materials with desired viscoelastic properties.
\end{abstract}

\section{Introduction}

Thermoplastic elastomers (TPEs) exhibit the properties and performance of cross-linked rubber but can be processed as thermoplastics. They are low modulus, flexible materials that can be stretched repeatedly to at least twice their original length at room temperature and return approximately to their initial length and shape upon stress release. The typical design of TPEs is based upon an ABA triblock copolymer architecture with the hard phase formed by component $\mathrm{A}$ (which behaves as a physical crosslinker), dispersed in the soft rubbery phase of component B. Most common TPEs include polystyrene-poly(butadiene)-polystyrene (SBS) (e.g. Styroflex®) and polystyrenepoly(isoprene)-polystyrene (SIS) (e.g. Kraton ${ }^{\circledR}$ D SIS series) which are widely used in combinations with other ingredients

${ }^{a}$ Department of Chemistry, University of Tennessee, Knoxville, TN, 37996, USA

${ }^{b}$ Chemical Sciences Division, Oak Ridge National Laboratory, Oak Ridge, TN, 37831, USA.E-mail: saitot@ornl.gov

${ }^{c}$ Center for Nanophase Materials Sciences, Oak Ridge National Laboratory, Oak Ridge, TN, 37831, USA

$\dagger$ This manuscript has been authored by UT-Battelle, LLC under Contract No. DE-AC05-00OR22725 with the U. S. Department of Energy. The publisher, by accepting the article for publication, acknowledges that the United States Government retains a non-exclusive, paid-up, irrevocable, worldwide license to publish or reproduce the published form of this manuscript, or allow others to do so, for United States Government purposes. The Department of Energy will provide public access to these results of federally sponsored research in accordance with the DOE Public Access Plan (http://energy.gov/downloads/doe-public-access-plan).

\$ Electronic supplementary information (ESI) available. See DOI: 10.1039/c7ra08641e (paraffinic oils, polyolefins, tackifying resins) in applications such as tire treads, adhesives, sealants, paving, footwear, packaging and many others. ${ }^{1-4}$

Multigraft copolymers ${ }^{5-7}$ with a rubbery backbone and glassy side chains have emerged as a new class of TPEs, so-called superelastomers, with superior elongation at break and low residual strains as compared to conventional TPEs. Mays et al. synthesized a series of well-defined poly(isoprene- $g$-styrene) multigraft copolymers with trifunctional, tetrafunctional and hexafunctional branch points. ${ }^{8}$ These studies showed that topology of the multigraft copolymers and the number of junction points per molecule can be two major parameters for adjusting their mechanical properties. ${ }^{9-13}$

The use of diene-based TPEs is, however, limited by the poor oxidation and UV resistance of the polydiene backbone and the relatively low upper service temperature $\left(<100{ }^{\circ} \mathrm{C}\right)$ in relation to the glass transition temperature of polystyrene. Efforts have been made to overcome these drawbacks by substituting the polydiene block with acrylates, such as poly( $n$-butyl acrylate) (PnBA) and other poly(alkyl acrylates), and the polystyrene block with methacrylates, such as poly(methyl methacrylate) $(\mathrm{PMMA})^{14,15}$ but the ultimate tensile properties of these triblock copolymers were poor as compared to the traditional dienebased TPEs. Depending on the alkyl substituent of the ester groups in polyacrylates and polymethacrylates, glass transition temperature can be tuned within a much larger temperature range (e.g. from $-89^{\circ} \mathrm{C}$ for poly $\left(n\right.$-nonyl acrylate) up to $259^{\circ} \mathrm{C}$ for poly(adamantyl methacrylate)). ${ }^{16}$ Furthermore, synthesizing multigraft superelastomers via a macromonomer approach using free radical or emulsion polymerization is compatible 
with these acrylic monomers, reduces the polymerization time, and minimizes the complexity of the synthetic route. Recently developed procedures allowed for the synthesis of PS- $g$-PI and all-acrylic multigraft copolymers showing very good thermal and mechanical properties. ${ }^{17-20}$ The macromonomer approach is a very useful method to synthesize multigraft copolymers since it readily controls the molecular weight and polydispersity of the side chains as well as the desired average number of branch points. ${ }^{21}$ The combination of this method with free radical polymerization provides a scalable and facile polymerization procedure with relatively good control of the molecular characteristics of the final multigraft copolymers.

In an effort to also improve the tensile strength and the thermal stability of thermoplastic elastomers, postpolymerization modifications on hydrogenated SBS and SIS copolymers have been widely performed. ${ }^{22-37}$ Sulfonation of the polystyrene block is a very versatile chemical modification that has been utilized not only to enhance microphase separation but also to mechanically reinforce the final material by introducing ionic interactions within the polymer chain networks. Sulfonation results in a significant increase of the Flory-
Huggins $\chi$ value and it has been $\operatorname{shown}^{27,28}$ that even low sulfonation levels can improve or alter microphase separation. Moreover, there is a critical value of sulfonation level (approximately $15-20 \%$ depending on the MW of the PS block), ${ }^{30,31}$ above which the polymer becomes non-thermally processable, poorlysoluble in most commonly used solvents and very brittle due to the highly ionically cross-linked networks. Thus, for a thermoplastic multigraft superelastomer, it is crucial to control the degree of sulfonation and find the fine line between increased tensile strength, high elongation, elasticity, and processability.

This study describes the synthesis and characterization of polystyrene- $g$-poly( $n$-butylacrylate) multigraft copolymers (abbreviated as PSm-PnBA) comprising of PnBA backbones and lightly sulfonated PS side chains synthesized by a macromonomer approach. The PS macromonomer (PSm) was synthesized by living anionic polymerization and high vacuum techniques, ${ }^{38}$ while conventional free radical polymerization was employed for the synthesis of the final multigraft copolymers (Scheme 1). The effects of the molecular weight, number of branch points and volume fraction on the properties of the final materials were investigated.

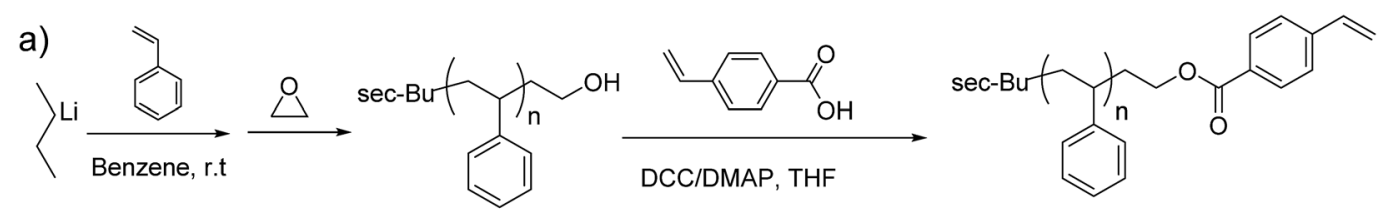

Hydroxyl terminated PS PS

Macromonomer

b)

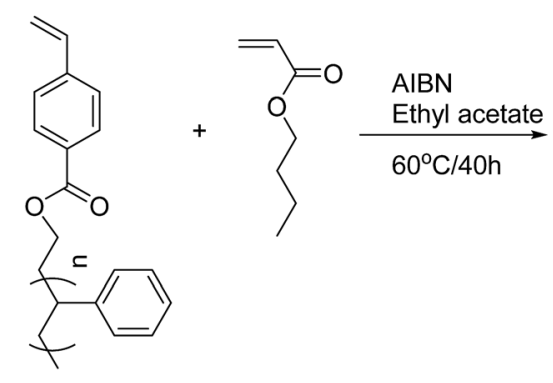

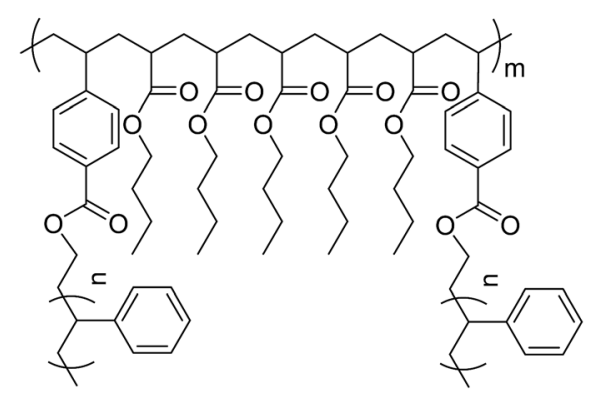

$60^{\circ} \mathrm{C} / 5 \mathrm{~h}$

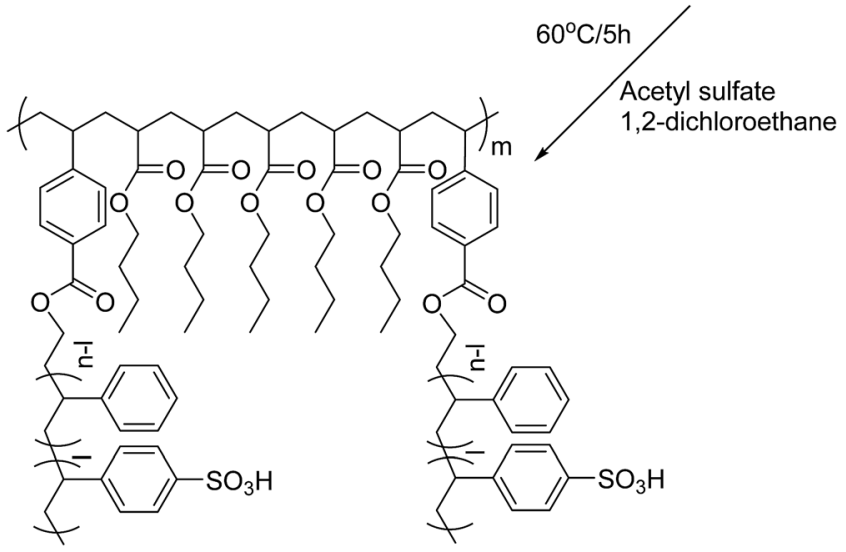

Scheme 1 (a) Synthesis of the PS macromonomer and (b) synthetic route and post-polymerization sulfonation of the multigraft copolymers. 


\section{Experimental}

Materials

The PS macromonomer was synthesized in evacuated, $n$-butyllithium washed and benzene rinsed glass vessels. Detailed descriptions of the high-vacuum technique as well as the purification procedures for styrene (Sigma Aldrich, $\geq 99 \%$ ), benzene (Sigma Aldrich, $\geq 99 \%$ ) and sec-BuLi (initiator, Acros, 1.3 M in cyclohexane/hexane 92/8) required for anionic polymerization have already been reported in the literature. ${ }^{38} 2,2^{\prime}$-Azobisisobutyronitrile (AIBN) (Sigma Aldrich, 98\%) was purified by crystallization from methanol, while an activated alumina (Sigma Aldrich, basic) column was utilized to remove the inhibitors from toluene (solvent, Fisher Chemical) and $n$-butyl acrylate ( $n$ BA) (Sigma Aldrich, $\geq 99 \%$ ). $N, N^{\prime}$-Dicyclohexylcarbodiimide (DCC) (Sigma Aldrich, 99\%), 4-(dimethylamino) pyridine (DMAP) (Sigma Aldrich, $\geq 99 \%$ ), 4-vinylbenzoic acid (Sigma Aldrich, 97\%), dichloroethane (DCE) (Fisher Chemical), acetic anhydride (Sigma Aldrich, $\geq 99 \%$ ) and sulfuric acid (Sigma Aldrich, 95-98\%) were used as received.

\section{Macromonomer synthesis}

The synthetic procedure for the PS macromonomer is shown in Scheme 1a. In an $n$-butyllithium/benzene rinsed glass apparatus equipped with break-seals, $40 \mathrm{~g}$ of styrene were introduced into $400 \mathrm{ml}$ of benzene solution with $4 \mathrm{mmol}$ of sec-butyllithium as initiator under high vacuum and the polymerization was left to proceed in room temperature. After 16 hours, $1.5 \mathrm{ml}$ of ethylene oxide were added to the living polystyrene anion solution and the color turned from orange to transparent within $1 \mathrm{~min}$. The reaction was kept at room temperature for 24 hours and was terminated with high vacuum sealed $\mathrm{MeOH}$. The resulting hydroxyl terminated polystyrene (PS-OH) was precipitated three times in large excess of $\mathrm{MeOH}$ and dried under reduced pressure at $40{ }^{\circ} \mathrm{C}$ for 48 hours. $39.3 \mathrm{~g}$ of $\mathrm{PS}-\mathrm{OH}$ were recovered and dissolved into $400 \mathrm{ml}$ of anhydrous THF with $16 \mathrm{mmol}$ of DCC and $16 \mathrm{mmol}$ of DMAP. A $10 \mathrm{ml}$ THF solution with $16 \mathrm{mmol}$ of 4 -vinylbenzoic acid were added to the solution and it was kept at room temperature. After 6 days, the polymer solution was precipitated in excess of $\mathrm{MeOH}$ three times in order to remove any excess of 4-vinylbenzoic acid and catalyst. The resulting PS macromonomer with polymerizable styrene end group was dried under reduced pressure at $40{ }^{\circ} \mathrm{C}$ for 48 hours and characterized with MALDI-TOFF.

\section{Multigraft copolymer synthesis and sulfonation}

All copolymerizations were conducted in high vacuum sealed round bottom flasks at $60{ }^{\circ} \mathrm{C}$ in toluene using AIBN as initiator. The PS macromonomer was always the minority component while the amount of initiator was tailored depending on the desirable final molecular weight and number of branching points. More specifically, for the synthesis of PSm-PnBA-1 multigraft copolymer, $0.651 \mathrm{~g}$ of PS macromonomer, $3.52 \mathrm{~g}$ of $n$-BA and $22 \mathrm{mg}$ of AIBN $(0.134 \mathrm{mmol})$ were introduced in a round bottom flask containing $30 \mathrm{ml}$ of toluene and were degassed in the high vacuum line twice. Then, the solution was put in an oil bath at $60{ }^{\circ} \mathrm{C}$ and the polymerization was left to proceed for 40 hours. After the completion of the polymerization, the flask was put in an ice bath and precipitated in $200 \mathrm{ml}$ of $\mathrm{MeOH}$, filtered and dried in a vacuum oven for 2 days. For the sulfonation procedure, $0.72 \mathrm{~g}$ of the dried PSm-PnBA-1 multigraft copolymer and $15 \mathrm{ml}$ of DCE were added in a round bottom flask exhibiting a side arm sealed with a rubber septum. The flask was attached in the high vacuum line, degassed twice, removed from the high vacuum line and placed in an oil bath at $60{ }^{\circ} \mathrm{C}$. A solution of $15 \mathrm{ml}$ of DCE, $0.113 \mathrm{ml}\left(1.2 \times 10^{-3} \mathrm{~mol}\right)$ of acetic anhydride and $0.03 \mathrm{ml}\left(6 \times 10^{-4} \mathrm{~mol}\right)$ of sulfuric acid was mixed and left to react for 10 minutes at $0{ }^{\circ} \mathrm{C}$. The mixture was then introduced with a syringe into the flask containing the PSm-PnBA-1 sample and the reaction was allowed to proceed for 5 hours under stirring at $60^{\circ} \mathrm{C}$ (change of color solution to light brown). $\mathrm{MeOH}$ was added to terminate the reaction and the final sulfonated multigraft copolymer was precipitated in excess of $\mathrm{MeOH}$, filtered, washed thoroughly with $\mathrm{DI}_{2} \mathrm{O}$ and $\mathrm{MeOH}$ three times, dried in a vacuum oven for 3 days and stored at $-20{ }^{\circ} \mathrm{C}$. Both the synthesis and sulfonation procedures for the multigraft copolymers are shown in Scheme $1 \mathrm{~b}$.

\section{Instrumentation}

\section{Molecular characterization}

Size exclusion chromatography (SEC) was carried out at $40{ }^{\circ} \mathrm{C}$ using a Polymer Laboratories GPC-120 unit equipped with a Precision Detectors PD 2040 (two-angle static light scattering (TALLS) detector), a Viscotek 220 differential viscometer and a Polymer Laboratories differential refractometer. A Polymer Laboratories PLgel; $7.5 \times 300 \mathrm{~mm} ; 10 \mu ; 500,10^{3}, 10 \times 10^{5}$ and $10 \times 10^{6} \AA$ column set was employed and the calibration range was 600-7 $500000 \mathrm{~g} \mathrm{~mol}^{-1}$ using PS standards. The mobile phase was THF at a flow rate of $1.0 \mathrm{ml} \mathrm{min}{ }^{-1} .{ }^{1} \mathrm{H}-\mathrm{NMR}$ was carried out on a Varian Mercury 500 instrument. All samples were dissolved in deuterated chloroform $\left(\mathrm{CDCl}_{3}\right)$.

\section{Thermal analysis}

Differential scanning calorimetry (DSC) was used to investigate the thermal properties of the samples. A TA Instruments Q2000 was utilized in temperatures ranging from $-140{ }^{\circ} \mathrm{C}$ to $160{ }^{\circ} \mathrm{C}$ at a heating rate of $10^{\circ} \mathrm{C} \mathrm{min}{ }^{-1}$ with a 2 minute isothermal hold at the maximum and minimum temperatures. Reported glass transition temperatures are those measured on the second of two heating runs.

\section{Morphological characterization}

Small angle X-ray scattering (SAXS) measurements were carried out in transmission geometry on a three pinhole collimated system equipped with a Rigaku rotating anode $(\mathrm{CuK} \alpha$ radiation $\lambda=1.542 \AA$, operating at $4.2 \mathrm{~kW}$ ), an Osmic multilayer mirror for higher proton flux and a MARCCD 2D detector (average pixel size $78.7 \times 78.7 \mu \mathrm{m})$. The sample to detector distance was 1.72 $\mathrm{m}$ and calibration was carried out using Ag-Behenate as the standard. All patterns were background corrected and normalized with respect to thickness and subsequently were radially 
averaged yielding 1D SAXS patterns of $I(q) v s$. $q$. The setup of the instrument enabled a resolvable range of $0.05 \mathrm{~nm}^{-1} \leq q \leq$ $2 \mathrm{~nm}^{-1}$. The bulk films were prepared by solution casting the polymers from a non-selective solvent (toluene) for 4 days in a porcelain crucible and then by annealing them in a vacuum oven at $130{ }^{\circ} \mathrm{C}$ (above the $T_{\mathrm{g}}$ of PS) for 3 more days. All films were quenched into liquid nitrogen after they were removed from the annealing oven in order to "trap" their thermodynamic state.

\section{Extensional rheometry and dynamic mechanical analysis (DMA)}

Uniaxial extension experiments of the samples were carried out on an HR2 rheometer (TA Instruments) using the SER3 fixture while an RSA-G2 dynamic mechanical analyzer (TA Instruments) was used for complimentary temperature ramp experiments. The specimens for the tests were prepared by solutioncasting the polymers in a Teflon Petri dish. Rectangular sheetlike specimens were cut out from the film by a razor blade and removed from the Teflon surface with the aid of dry ice. All the uniaxial extension and DMA experiments were performed with constant Hencky strain rates.

\section{Results and discussion}

\section{Synthesis of lightly sulfonated multigraft acrylate-based copolymers}

The molecular characteristics of the PS macromonomer (PSm) as well as the multigraft superelastomers are presented in Table 1. The results show well-defined final products with narrow polydispersity for PSm (1.03) and controlled molecular weight, $\bar{M}_{\mathrm{n}}=$ $10300 \mathrm{~g} \mathrm{~mol}^{-1}$ (Fig. S1 $\$$ ). The PS macromonomer serves as the PS side chain of the multigraft copolymers. The free radical copolymerization of PS macromonomer with $n$-butyl acrylate at high monomer concentrations with an appropriate molar ratio of AIBN (4 mg of initiator per one $\mathrm{ml}$ of monomer) resulted in controlled molecular weights and number of branches for the final copolymers. Higher amounts of AIBN and solvent produced much shorter polymer chains for the $n$-butyl acrylate backbone, thus lower number of branches and lower molecular weights for the branched copolymers. Consequently, it was crucial to regulate the amount of AIBN needed, in order to achieve highly branched copolymers and high conversions of the monomers. Although the exact targeted final molecular weights for the multigraft copolymers are difficult to achieve using free radical polymerization, we were able to achieve final molecular weights with values very close
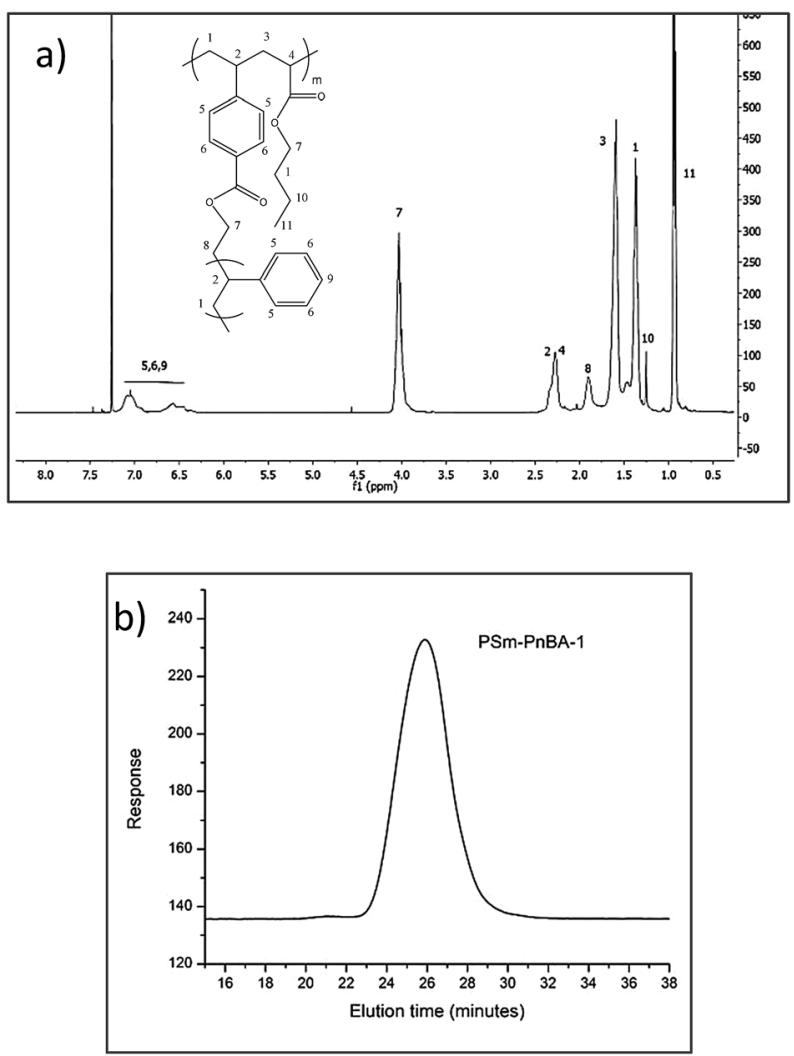

Fig. 1 (a) ${ }^{1} \mathrm{H}-\mathrm{NMR}$ spectrum and (b) SEC chromatogram of the PSmPnBA-1 multigraft copolymer.

to the targeted theoretical molecular weights by adjusting the appropriate ratio of AIBN/n-butyl acrylate/solvent.

${ }^{1} \mathrm{H}-\mathrm{NMR}$ in combination with SEC (Fig. 1a and b respectively) was used to characterize the molecular weights of the final copolymers, their PS content, the average number of branch points and the sulfonation degree. The content of PS glassy side chains was kept at $\sim 20$ wt $\%$ since previous studies ${ }^{9-13}$ showed that the highest modulus with the highest elongation rates were obtained with a glassy side chain composition of approximately $20 \mathrm{wt} \%$. The sulfonated derivatives of the two PSm-PnBA multigraft copolymers are labelled as PSSm-PnBA followed by their respective numbers ( 1 and 2 ).

Fig. 1b shows the SEC chromatogram corresponding to PSmPnBA-1 multigraft copolymer exhibiting a monomodal peak. The absence of any other peaks in lower molecular weights verifies that there is no unreacted PS macromonomer present in the final multigraft copolymer. The relative ${ }^{1} \mathrm{H}-\mathrm{NMR}$ (Fig. 1a)

Table 1 Molecular characterization results for the synthesized multigraft copolymers

\begin{tabular}{|c|c|c|c|c|c|c|}
\hline Sample & $\begin{array}{l}M_{\mathrm{n}}^{\mathrm{PS} m a c r o} \\
\left(\mathrm{~kg} \mathrm{~mol}{ }^{-1}\right)\end{array}$ & $\begin{array}{l}M_{\mathrm{n}}^{\text {total } a} \\
\left(\mathrm{~kg} \mathrm{~mol}^{-1}\right)\end{array}$ & PDI & $\begin{array}{l}\text { PS weight } \\
\text { fraction } f\end{array}$ & $\begin{array}{l}\text { Number of } \\
\text { branch points }\end{array}$ & $\begin{array}{l}\text { Sulfonation } \\
\text { degree }^{b}(\mathrm{~mol} \%)\end{array}$ \\
\hline PSm-PnBA-1 & 10.3 & 195.0 & 1.75 & 0.20 & 4 & 13.5 \\
\hline PSm-PnBA-2 & 10.3 & 125.4 & 1.80 & 0.20 & 3 & 13.5 \\
\hline
\end{tabular}


peaks corresponding to PS aromatic rings (6.3-7.3 ppm) and to PnBA backbone acrylate group (methylene next to ester, $4 \mathrm{ppm}$ ) respectively, confirm the successful random copolymerization of the monomers.

In order to calculate the branching points of the multigraft copolymers, we used the final molecular weight of each multigraft copolymer, the "fixed" molecular weight of the PS macromonomer and the final weight fraction of each component calculated by ${ }^{1} \mathrm{H}-\mathrm{NMR}$ spectroscopy. For both multigraft copolymers, the PS weight fraction is $20 \%$, thus the total molecular weight of PS content is $195 \mathrm{k} \times 20 / 100=39 \mathrm{k}$ and $125.4 \mathrm{k} \times 20 / 100=25.08 \mathrm{k}$ for PSm-PnBA-1 and PSm-PnBA-2 respectively. By dividing the total molecular weight of PS content for each of the two multigraft copolymers with the molecular weight of the PS macromonomer, we get the average number of branching points for PSm-PnBA-1 and PSm-PnBA-2 respectively. This indicates that PSm-PnBA-1 sample exhibits mostly 4 branching points per chain while PSm-PnBA-2 exhibits 2 and 3 branching points per chain.

The sulfonation degree of PS side chains $(20 \%$ of the total molecular weight) was kept at $13.5 \%$ in order to maintain processability which gives ion exchange capacity (IEC) of $0.26 \mathrm{mmol} \mathrm{g}^{-1}$. During sulfonation, controlling the molar ratio of acetyl sulfate/styrene is very important for achieving the desired sulfonation level, and in this study the ratio was kept at 0.5 (i.e. [acetyl sulfate]/[styrene] $=0.5$ ). The sulfonation level of each multigraft copolymer (SL\%) was calculated by using ${ }^{1} \mathrm{H}$ NMR and the following equation: ${ }^{22}$

$$
\mathrm{SL} \%=\frac{\text { Integr. of arom. rings of sulf. copolymer }}{\text { Integr. of arom. rings of non-sulf. copolymer }}
$$

DSC measurements were performed for both PSm-PnBA-1 and PSm-PnBA-2 as well as for their relative sulfonated samples PSSm-PnBA-1 and PSSm-PnBA-2. For all samples, only one glass transition temperature was observed (Fig. S2a and bł). This suggests that the rapid cooling rate in DSC did not provide sufficient time for microphase separation to occur, which is consistent with observed results in other experiments with similar graft copolymers. ${ }^{39,40}$ Since poly( $n$-butyl acrylate) is the majority component, the glass transition temperature is shifted towards lower temperatures and closer to the $T_{\mathrm{g}}$ of PnBA. Sulfonation of the PS block shifts the $T_{\mathrm{g}}$ towards higher temperatures, increasing it from $-46{ }^{\circ} \mathrm{C}$ for PSm-PnBA-1 to $-40{ }^{\circ} \mathrm{C}$ for PSSm-PnBA-1 (sulfonated) and from $-42{ }^{\circ} \mathrm{C}$ for PSmPnBA-2 to $-39{ }^{\circ} \mathrm{C}$ for PSSm-PnBA-2 (sulfonated).

The increase of $T_{\mathrm{g}}$ is associated with the ion content and the ionomeric effect which is caused by regions with restricted mobility that act as physical cross-links. ${ }^{\mathbf{4 0 , 4 1}}$ The $T_{\mathrm{g}}$ of both nonsulfonated samples is slightly lower than expected, which can be possibly attributed to small traces of solvent trapped in the copolymer, even though the samples were dried thoroughly. Also from Fig. S2a and b, + it is observed that the curve indicating the glass transition, occurs over a relatively broad range of temperatures and thus, it may introduce an error of $\pm 2-3{ }^{\circ} \mathrm{C}$ in the measurements.

\section{Viscoelastic properties}

Extensional rheometry (SER3) at room temperature was used to determine the non-linear viscoelastic properties of the multigraft copolymers and to investigate the impact of sulfonation on these properties. The linear viscoelastic characterization was performed by small amplitude oscillatory shear.

Sulfonation has a significant impact on the linear viscoelastic response for PSm-PnBA-1 and PSm-PnBA-2 (Fig. 2a and b). In the case of PSm-PnBA-1, storage and loss modulus are increased by approximately two orders of magnitude at room temperature after sulfonation. The change of slope is due to the change from liquid-like to solid-like behavior after sulfonation. The crossover for the PSm-PnBA-1 before sulfonation is associated with the relaxation behavior of the system and the sample is liquid-like at low frequencies and solid-like at high frequencies. For PSmPnBA-2, $G^{\prime}$ and $G^{\prime \prime}$ increase by one order of magnitude with sulfonation. Before sulfonation, the loss and storage modulus of PSm-PnBA-1 are lower than those of PSm-PnBA-2 which is caused by the longer distance among the PS side chains and the less dense packing of those chains due to the higher molecular weight of the PnBA backbone of the PSm-PnBA-1 multigraft copolymer. On the other hand, after sulfonation, the loss and storage modulus of PSSm-PnBA-1 are much higher than those of PSSmPnBA-2 since there are more sulfonated PS side chains per macromolecule in PSSm-PnBA-1. The sulfonated PS chains act as physical crosslinks forming ionic bonds between the pendant
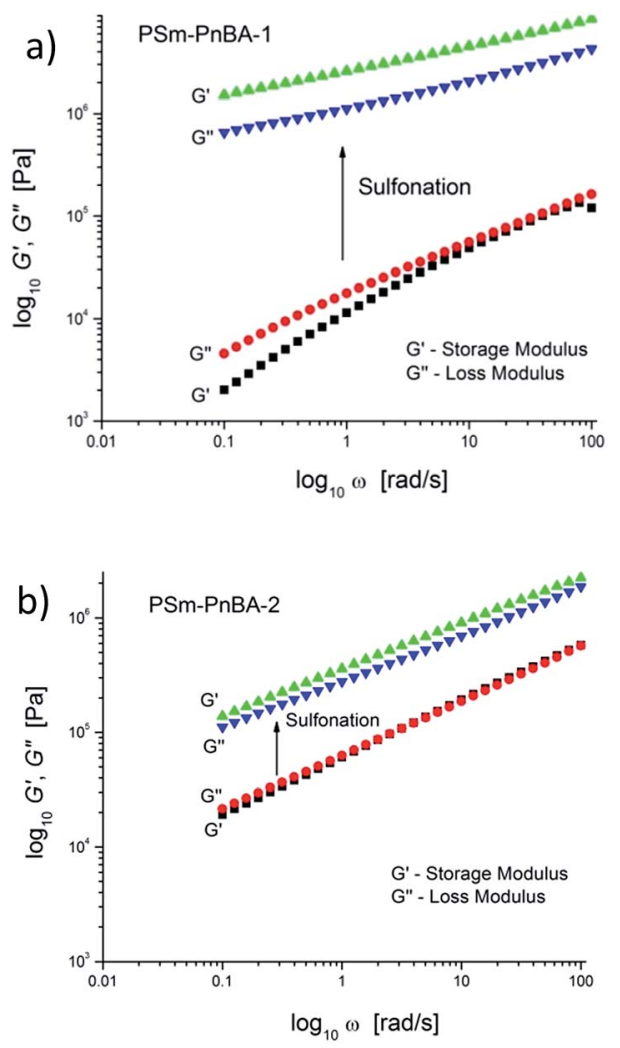

Fig. 2 Linear viscoelastic data in room temperature for (a) PSm-PnBA1 and (b) PSm-PnBA-2 copolymers before and after sulfonation. Storage and loss modulus increase drastically after sulfonation for both samples. 
$-\mathrm{SO}_{3}{ }^{-} \mathrm{H}^{+}$groups which dominate the dynamics of both systems. Increasing the number of branching points only by 1 (from 3 to 4 ) storage and loss modulus increase drastically.

In a recent study, ${ }^{42}$ Filippidi et al. investigated a similar mechanism where they introduced strong iron charges $\left(\mathrm{Fe}^{3+}\right)$ into a loosely cross-linked amorphous epoxy network with high catechol content in order to form dynamic iron-catechol cross-links. They showed that this mussel inspired system enhances stiffness and toughness without trading off elasticity, due to the incorporation of ionomeric nanodomains into a cross-linked network. The non-linear viscoelastic measurements for PSm-PnBA-1 and PSSm-PnBA-1 copolymers were conducted at three different strain rates $0.1,1$ and $10 \mathrm{~s}^{-1}$ (Fig. S3a and b:). The true stress is significantly increased after sulfonation for all strain rates without sacrificing elongation (true strain) very much. Fig. 3 shows the notable increase in true stress after sulfonation for each one of the three strain rates measured. For $0.1 \mathrm{~s}^{-1}$ (Fig. 3a), true stress for PSm-PnBA1 is $0.365 \mathrm{MPa}$ while after sulfonation true stress reaches 10.6 MPa for the same true strain value $(\varepsilon=2)$. Both PSmPnBA-1 and PSSm-PnBA-1 (sulfonated) samples follow the same trend for the rest of the strain rates (Fig. $3 \mathrm{~b}$ and c). True stress increases from 1.74 MPa to 33.9 MPa and from 5.86 MPa to $84.9 \mathrm{MPa}$ for $1 \mathrm{~s}^{-1}$ and $10 \mathrm{~s}^{-1}$ respectively for $\varepsilon=2$. The elongation (true strain) at break before sulfonation for PSm-PnBA-1 was at 2060\% $(\varepsilon=3)$ for strain rates $1 \mathrm{~s}^{-1}$ and $10 \mathrm{~s}^{-1}$ and at $1380 \%(\varepsilon=2.6)$ with strain rate equal to $0.1 \mathrm{~s}^{-1}$, while after sulfonation the elongation at break was decreased (but still remained significant) to $\sim 700 \%(\varepsilon=2)$ for all three strain rates tested. Similar behavior is observed for the PSmPnBA-2 sample and its sulfonated derivative PSSm-PnBA-2 where true stress increases as the strain rate increases for both samples (Fig. S4a and b\$). On the other hand, elongation (true strain) at break for both PSm-PnBA-2 and PSSm-PnBA-2 remains similar for all strain rates tested. Fig. 4 shows the increase in true stress after sulfonation for all three different strain rates. The low degree of sulfonation does not alternate significantly the elongation of both PSm-PnBA-2 and PSSmPnBA-2 and the materials maintain their elasticity while true stress increases 2 to 4 times more than the non-sulfonated case. For $0.1 \mathrm{~s}^{-1}$ (Fig. 4a), true stress for PSm-PnBA-2 is 1.71 MPa while after sulfonation true stress reaches $4.1 \mathrm{MPa}$ for the same true strain value $(\varepsilon=2.2)$. Both PSm-PnBA-2 and PSSm-PnBA-2 samples follow the same trend for the rest of the strain rates (Fig. 4b and c), where the true stress increases from 4.8 MPa to 15.1 MPa and from 16.2 MPa to 36.1 MPa for 1 $\mathrm{s}^{-1}$ and $10 \mathrm{~s}^{-1}$ respectively. Sulfonation has a greater impact on the non-linear viscoelastic properties of PSm-PnBA-1 than those of PSm-PnBA-2, which can be explained by the fact that the PS side chains of PSm-PnBA-1 form more ionic bonds per macromolecule as compared to the lower average numbers of $-\mathrm{SO}_{3}{ }^{-} \mathrm{H}^{+}$groups in PSm-PnBA-2. Sulfonating one more PS branch in combination with the increase of the total molecular weight of the PSm-PnBA-1 multigraft copolymer, gives a much higher increase of true stress after sulfonation as compared to PSm-PnBA-2. This increase in true stress for both PSSm-PnBA-1 and PSSm-PnBA-2 multigraft copolymers
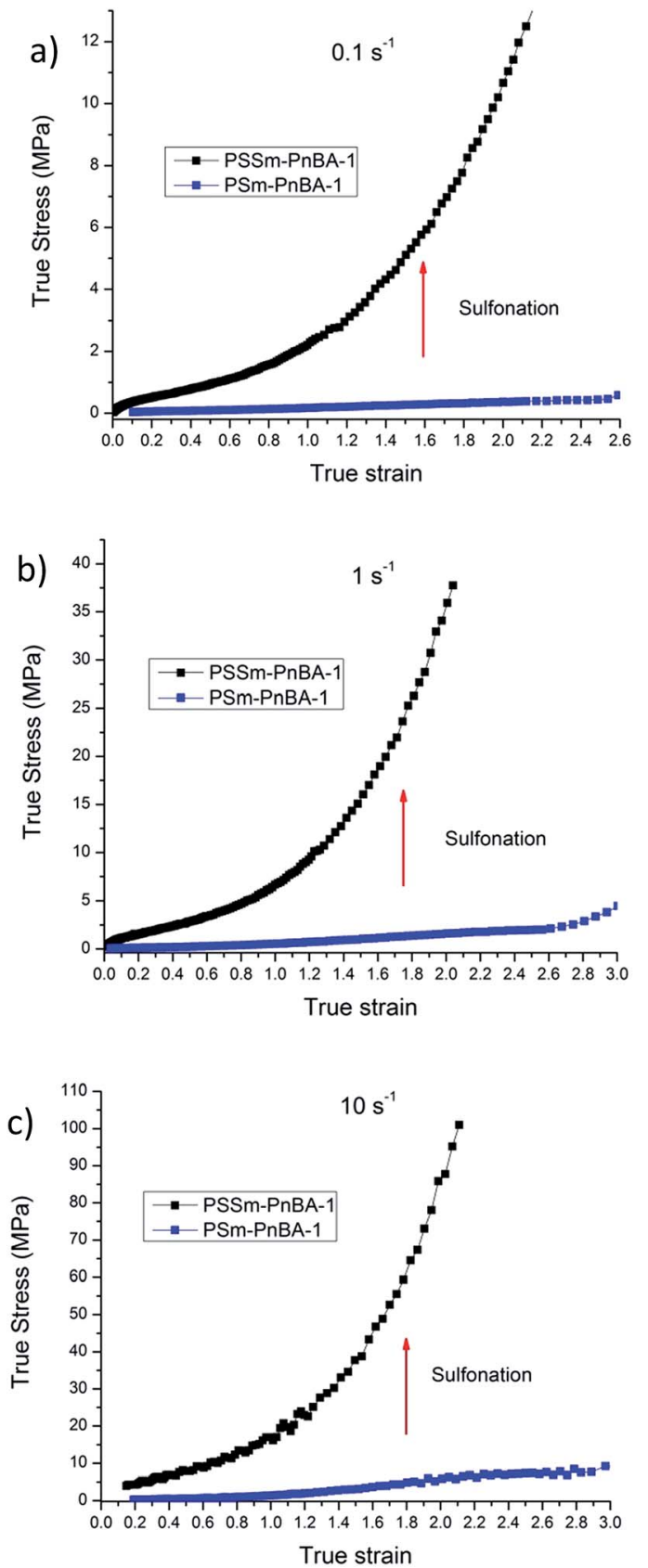

Fig. 3 Non-linear viscoelastic data comparison between PSm-PnBA-1 and PSSm-PnBA-1 for (a) $0.1 \mathrm{~s}^{-1}$ (b) $1 \mathrm{~s}^{-1}$ (c) $10 \mathrm{~s}^{-1}$.

indicates the significant impact on the viscoelastic properties caused by sulfonating only a very small fraction $(2-3 \%$, IEC $\sim 0.26 \mathrm{mmol} \mathrm{g}^{-1}$ ) of the final material (i.e. sulfonating $13.5 \mathrm{wt} \%$ of the PS chains which occupy $20 \mathrm{wt} \%$ of the whole multigraft copolymer). Slightly altering the properties of the glassy side chains, i.e. tuning the PS chemical structure by adding $-\mathrm{SO}_{3}{ }^{-} \mathrm{H}^{+}$groups that can form ionic bonds, and changing the number of branching points of the copolymers will allow one to take advantage of the favourable branched architecture and manipulate the thermoplastic and elastic properties of the copolymer towards a desired direction. 
a)
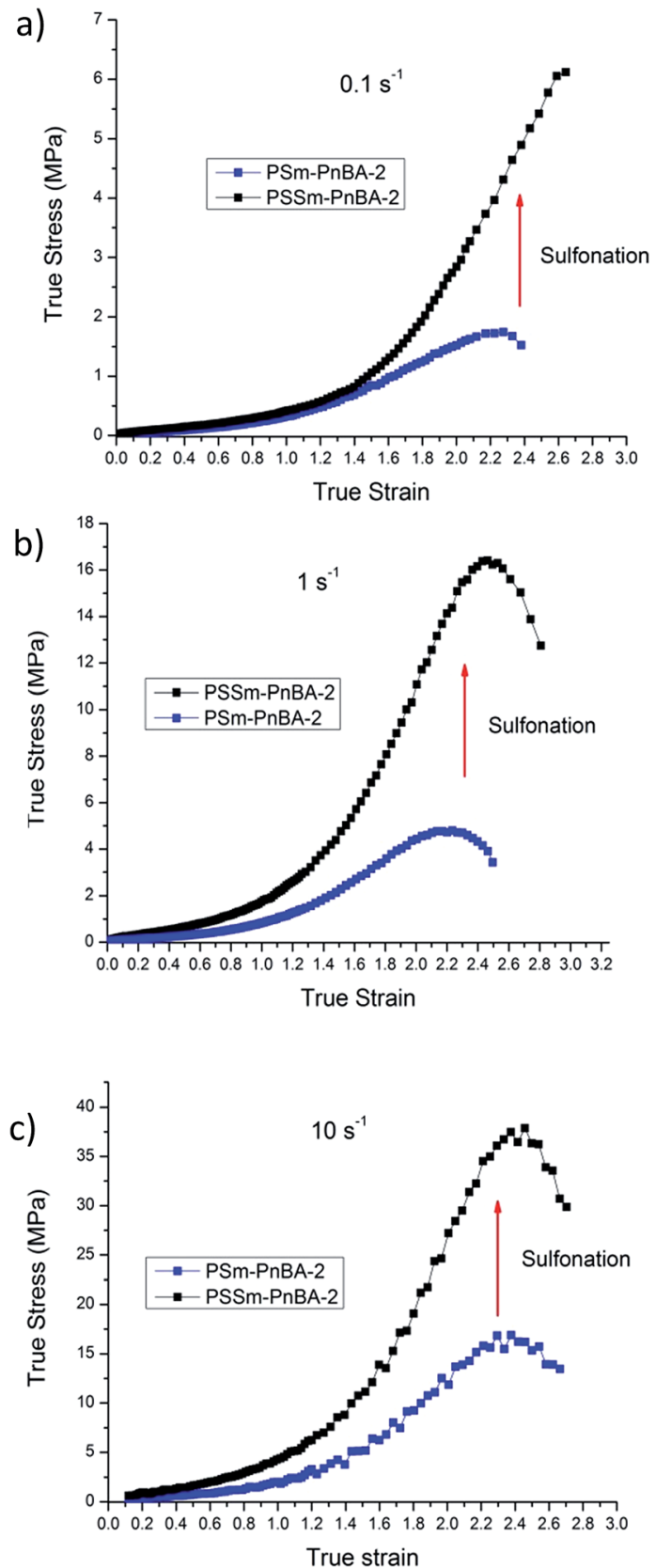

Fig. 4 Non-linear viscoelastic data comparison between PSm-PnBA2 and PSSm-PnBA-2 for (a) $0.1 \mathrm{~s}^{-1}$ (b) $1 \mathrm{~s}^{-1}$ (c) $10 \mathrm{~s}^{-1}$.

\section{Morphology}

Fig. 5 shows the SAXS patterns of PSm-PnBA-1, PSSm-PnBA-1, PSm-PNBA-2 and PSSm-PnBA-2 (Fig. 5a-d respectively). All samples (non-sulfonated and sulfonated) were annealed at $130{ }^{\circ} \mathrm{C}$ and they appear to exhibit no long range order since no scattering peaks are observed. In all cases, the total volume fraction of PS block is $\Phi_{\mathrm{PS}}=0.19$ (calculated from the equation $\Phi_{\mathrm{A}}=f_{\mathrm{A}} d_{\mathrm{A}} /\left(f_{\mathrm{A}} d_{\mathrm{A}}+f_{\mathrm{B}} d_{\mathrm{B}}\right)$, where $d_{\mathrm{A}}=d_{\mathrm{PS}}=1.05 \mathrm{~g} \mathrm{ml}^{-1}$ and $d_{\mathrm{B}}=$ $d_{\mathrm{PnBA}}=1.087 \mathrm{~g} \mathrm{ml}^{-1}$ and $f_{\mathrm{A}}=0.2$ and $f_{\mathrm{B}}=0.8$ are the weight fractions of PS and PnBA, respectively, determined by ${ }^{1} \mathrm{H}-\mathrm{NMR}$ spectroscopy). It is well-known ${ }^{43}$ that the product of the
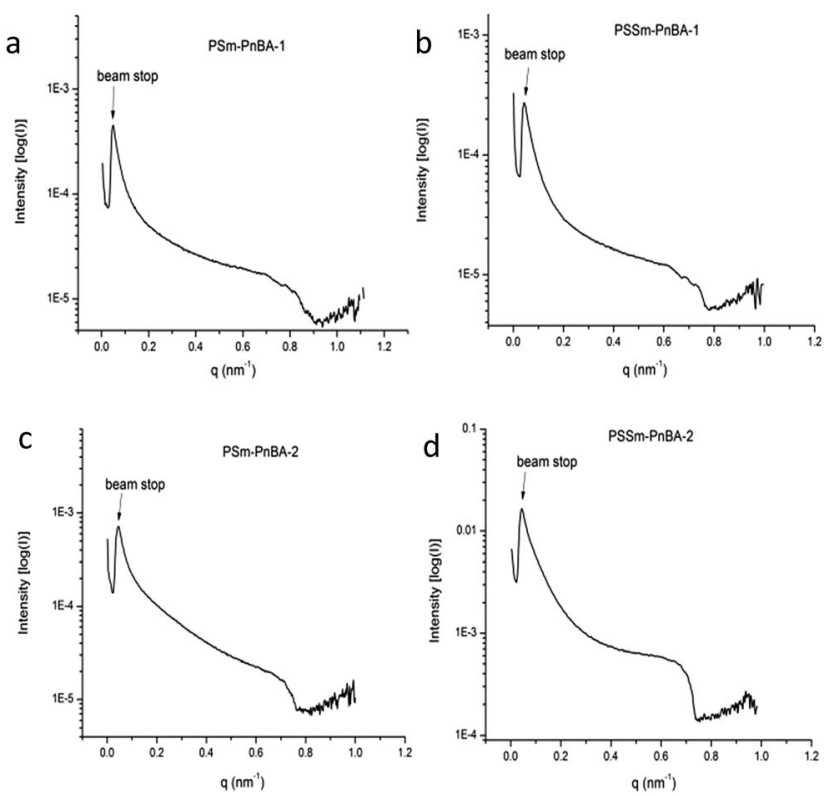

Fig. 5 SAXS data of (a) PSm-PnBA-1 (b) PSSm-PnBA-1 (c) PSm-PnBA2 and (d) PSSm-PnBA-2 multigraft copolymers.

Florry-Huggins interaction parameter $\chi$ with the degree of polymerization $N$ and in combination with the volume fraction $\Phi$ can provide information about the predicted morphology of a given block copolymer. The values of $\chi_{\mathrm{AB}}$ for a block copolymer with A and B blocks can be estimated by the molar volumes and the solubility parameters of the two components via eqn (2):

$$
\chi_{\mathrm{AB}}=\frac{V_{\mathrm{m}} \times\left|\delta_{\mathrm{A}}-\delta_{\mathrm{B}}\right|^{2}}{R T}
$$

where $V_{\mathrm{m}}$ is the total molar volume, $\delta_{\mathrm{A}}$ and $\delta_{\mathrm{B}}$ are the solubility parameters of PS and PnBA respectively, $R$ the gas constant equal to $8.314\left(\mathrm{~J} \mathrm{~K}^{-1} \mathrm{~mol}^{-1}\right)$ and $T$ the temperature $(298 \mathrm{~K})$. The molar volumes of PS, PnBA and PSS are 99.2, 118.7 and $103.2\left(\mathrm{~cm}^{3} \mathrm{~mol}^{-1}\right)$ respectively while the solubility parameters for PS, PnBA and PSS are 18.66, 18.5 and $19.26\left(\mathrm{~J} \mathrm{~cm}^{-3}\right)^{1 / 2}$ respectively. ${ }^{40,44}$ The above numbers of molar volume and solubility parameter for the PSS segment correspond to $10 \%$ sulfonation, ${ }^{45}$ which is very similar to the degree of sulfonation in this study. The total molar volume $V_{\mathrm{m}}$ is given by the expression $\left(V_{\mathrm{A}} \times V_{\mathrm{B}}\right)^{1 / 2}$, where $V_{\mathrm{A}}$ and $V_{\mathrm{B}}$ are the molar volumes of PS and PnBA respectively. Furthermore, the total degree of polymerization $N$ for the multigraft copolymers is given by the following equation:

$$
N^{\text {multi }}=\frac{N^{\text {tot }}}{l}
$$

where $l$ is the number of branches of the multigraft copolymer and $N^{\text {tot }}$ is the total degree of polymerization of the two components equal to $N^{\text {tot }}=N_{\mathrm{A}}+N_{\mathrm{B}}$, where $N_{\mathrm{A}}$ and $N_{\mathrm{B}}$ are the degrees of polymerization of PS and PnBA respectively. Table 2 summarizes the $\chi$ and $\chi N$ values calculated using eqn (2) and (3) for PSm-PnBA-1, PSm-PnBA-2, PSSm-PnBA-1 and PSSm-PnBA-2. It is evident that the Flory-Huggins interaction parameter for 
Table 2 Summary of $\chi N$ values for the synthesized multigraft copolymers

\begin{tabular}{|c|c|c|c|c|}
\hline Sample & $\Phi_{\mathrm{PS}}$ & Florry-Huggins interaction parameter $\chi$ & Degree of polymerization ${ }^{a} N^{\text {multi }}$ & $\chi N$ \\
\hline PSm-PnBA-1 & 19 & 0.0011 & 325 & 0.36 \\
\hline PSm-PnBA-2 & 19 & 0.0011 & 279 & 8.38 \\
\hline PSSm-PnBA-2 & 19 & 0.0258 & 279 & 7.19 \\
\hline
\end{tabular}

the PS/PnBA system is very low $\left(\chi_{\mathrm{PS} / \mathrm{PnBA}}=0.00111\right)$ while for the $\mathrm{PSS} / \mathrm{PnBA}$ system is increased due to the introduction of higher polarity through the sulfonated pendant groups $\left(\chi_{\mathrm{PSS} / \mathrm{PnBA}}=0.0258\right)$. However, the $\chi_{\mathrm{PSS} / \mathrm{PnBA}}$ value is still very low, which indicates that PS and PnBA should not phase separate in a long range order, even after sulfonating a small amount of polystyrene chains. The presence of the branches in such high molecular weight copolymers dramatically hinders reptation necessary for improved ordering. In addition, the randomness of the position of the branch points further reduces long range order. Our system is asymmetric, since it exhibits only $19 \%$ of polystyrene, and due to the grafting architecture, it follows different behavior than a symmetric linear diblock copolymer. According to theoretical studies ${ }^{46}$ using the reciprocal-space self-consistent field theory (SCFT), the phase diagrams of graft copolymers are highly asymmetric and the boundaries of order-order transitions shift due to the variations in the chain stretching energy. As a result, it is calculated that for $\chi N \leq 14$ and for $\Phi_{\mathrm{PS}} \leq 25$, no long range order is expected, which is in agreement with our experimental observations through SAXS. On the other hand, previous studies $^{47,48}$ on thermoplastic multigraft copolymers have shown that materials of this molecular weight and chemical composition should microphase separate, even though they do not exhibit long range order. In order to validate that, we conducted temperature ramp experiments from -100 to $160{ }^{\circ} \mathrm{C}$ using an RSA-G2 dynamic mechanical analyzer (TA Instruments) and an HR2 rheometer (TA Instruments) and the results of storage and loss modulus for PSm-PnBA-2 and PSSm-PnBA-2 are shown in Fig. S5 and 6.\$ At low temperatures $\left(-100\right.$ to $\left.20^{\circ} \mathrm{C}\right)$ and close to the $T_{\mathrm{g}}$ of PnBA, both non-sulfonated and sulfonated samples exhibit a very prominent change in their storage and loss modulus corresponding to the PnBA phase (Fig. S5a and $\mathrm{b}:$ respectively). This change shows that the soft PnBA segment undergoes a transition from the glassy state to the rubbery state, indicating that the multigraft copolymers are phase separated in soft and hard domains even though they do not exhibit any long range order. If the samples exhibited only one homogeneous phase, that slope change would not have been so intense and we would rather observe a smoother decrease of storage modulus over the whole temperature range tested rather than a vast change near the glass transition temperature of PnBA. As the temperature increases above $20{ }^{\circ} \mathrm{C}$, both PSm-PnBA-2 and PSSm-PnBA-2 show no significant observable change of the slope. The lack of a clear transition near the $T_{\mathrm{g}}$ of polystyrene is probably due to the low content of polystyrene hard segment in the copolymer. It rather suggests the presence of a weak phase separation by also considering that these graft copolymers flow near the $T_{\mathrm{g}}$ of polystyrene (Fig. S6a and bt respectively).

\section{Conclusions}

In conclusion, the concept of synthesizing a PS macromonomer (side chains) through anionic polymerization and then copolymerizing it with $n$-butyl acrylate using free radical polymerization proves to be very efficient since the strategy does not require tedious multi-step anionic polymerization or any fractionation or separation. The overall time for the synthesis of the multigraft copolymers with controlled molecular weight and number of branches was significantly reduced and the method could be readily adoptable in industry. Slight sulfonation of PS- $g$-PnBA multigraft superelastomers $(2-3 \mathrm{wt} \%$ total) shows that we can further improve their viscoelastic properties and increase true stress significantly, while maintaining high elongation, not only by tuning their molecular characteristics but also by altering their chemical structure. On the other hand sulfonation of PS side chains does not seem to have any significant impact on the morphology of the multigraft copolymers, which remain weakly microphase separated but do not exhibit any long range order.

\section{Conflicts of interest}

There are no conflicts to declare.

\section{Acknowledgements}

This work was supported by the U.S. Department of Energy, Office of Science, Basic Energy Sciences, Materials Sciences and Engineering Division. A portion of this research was conducted at the Center for Nanophase Materials Sciences, which is a DOE Office of Science User Facility.

\section{Notes and references}

1 R. J. Spontak and P. N. Patel, Curr. Opin. Colloid Interface Sci., 2000, 5, 334-341.

2 K. Knoll and N. Nießner, Macromol. Symp., 1998, 132, 231243.

3 R. N. Legge, Rubber Chem. Technol., 1989, 62, 529-547. 
4 R. N. Legge, G. Holden and E. H. Schröeder, Thermoplastic Elastomers - A Comprehensive Review, Hanser Publishers, Munich, 1987.

5 D. Uhrig and J. W. Mays, Polym. Chem., 2011, 2, 69-76.

6 N. Hadjichristidis, M. Pitsikalis, S. Pispas and H. Iatrou, Chem. Rev., 2001, 101, 3747-3792.

7 R. Schlegel, et al., Macromolecules, 2011, 44, 9374-9383.

8 D. Uhrig and J. W. Mays, Macromolecules, 2002, 35, 71827190.

9 Y. Zhu, et al., Macromolecules, 2006, 39, 4428-4436.

10 R. Schlegel, et al., Eur. Polym. J., 2009, 45, 2902-2912.

11 D. Uhrig, R. Schlegel, R. Weidisch and J. W. Mays, Eur. Polym. J., 2011, 47, 560-568.

12 U. Staudinger, et al., Eur. Polym. J., 2008, 44, 3790-3796.

13 R. Schlegel, et al., Polymer, 2009, 50, 6297-6304.

14 D. J. Tong and R. Jerome, Polymer, 2000, 41, 2499-2510.

15 D. J. Tong, et al., Polymer, 2001, 42, 3503-3514.

16 C. C. Cypcar, P. Camelio, V. Lazzeri, J. L. Mathias and

B. Waegell, Macromolecules, 1996, 29, 8954-8959.

17 A. Goodwin, et al., Ind. Eng. Chem. Res., 2015, 54, 9566-9576. 18 W. Wang, et al., Ind. Eng. Chem. Res., 2015, 54, 1292-1300.

19 H. Li, et al., J. Colloid Interface Sci., 2015, 453, 226-236.

20 Y. Luo, X. Wang, Y. Zhu, B.-C. Li and S. Zhu, Macromolecules, 2010, 43, 7472-7481.

21 X. Leng, et al., RSC Adv., 2016, 6(36), 30320-30329.

$22 \mathrm{~J}$. Yeo, et al., Nanotechnology, 2012, 245703, (11pp).

23 S. Kim, et al., J. Membr. Sci., 2013, 427, 85-91.

24 Y. A. Elabd and E. Napadenski, Polymer, 2004, 45, 3037-3043. 25 J.-E. Yang and J.-S. Lee, Electrochim. Acta, 2004, 50, 617-620. 26 Y. A. Elabd, E. Napadenski, J. M. Sloan, D. M. Crawford and

C. W. Walker, J. Membr. Sci., 2003, 217, 227-242.

27 Y. A. Elabd, E. Napadenski, C. W. Walker and K. I. Winey, Macromolecules, 2006, 39, 399-407.

28 X. Lu, W. P. Steckle and R. A. Weiss, Macromolecules, 1993, 26, 6525-6530.
29 A. Buonerba, V. Speranza and A. Grassi, Macromolecules, 2013, 46, 778-784.

30 R. A. Weiss, A. Sen, C. L. Willis and L. A. Pottick, Polymer, 1991, 32, 1867-1874.

31 R. A. Weiss, A. Sen, C. L. Willis and L. A. Pottick, Polymer, 1991, 32, 2785-2792.

32 J. Won, et al., J. Membr. Sci., 2003, 214, 245-257.

33 K. A. Mauritz, R. I. Blackwell and F. L. Beyer, Polymer, 2004, 45, 3001-3016.

34 B. Kim, J. Kim and B. Jung, J. Membr. Sci., 2005, 250, 175182.

35 E. A. Elabd, C. W. Walker and F. L. Beyer, J. Membr. Sci., 2004, 231, 181-188.

36 R. I. Blackwell and K. A. Mauritz, Polymer, 2004, 45, 34573463.

37 J. Kim, et al., Macromol. Rapid Commun., 2002, 23, 753-756.

38 D. Uhrig and J. W. Mays, J. Polym. Sci., Part A: Polym. Chem., 2005, 43, 6179-6222.

39 J. L. De La Fuente, M. Fernandez-Garcia and E. L. Madruca, J. Appl. Polym. Sci., 2001, 80, 783-789.

40 T. Saito, et al., Macromolecules, 2008, 41, 3503-3512.

41 A. Eisenberg, B. Hird and R. B. Moore, Macromolecules, 1990, 23, 4098-4107.

42 E. Filippidi, et al., Science, 2017, 358, 502-505.

43 L. Leibler, Macromolecules, 1980, 13, 1602-1617.

44 J. Brandrup, E. H. Immergut and E. A. Grulke, Polymer Handbook, Wiley-Interscience Publication, New York, 4th edn, 1999.

45 S. P. Carvallo, E. F. Lucas, G. Gonzales and L. S. Spinelli, J. Braz. Chem. Soc., 2013, 24, 1998-2007.

46 L. Zhang, J. Lin and s. Lin, J. Phys. Chem. B, 2008, 112, 97209728.

47 W. Wang, et al., Macromolecules, 2014, 47, 7284-7295.

48 J. Mijovic, M. Sun, S. Pejanovic and J. W. Mays, Macromolecules, 2003, 36, 7640-7651. 